\title{
Adhesion of Sputtered Nickel Films on Polycarbonate Substrates
}

\author{
Xueqiang Qian, Xiaolu Pang, Kewei Gao, Huisheng Yang, Jie Jin, and Alex A. Volinsky
}

\author{
(Submitted May 3, 2013; in revised form October 16, 2013; published online December 3, 2013)
}

\begin{abstract}
Nickel films were deposited by radio frequency magnetron sputtering on top of polycarbonate substrates. Surface energy of the substrate was measured by means of the contact angle technique. Effects of sputtering parameters on the critical load between the film and the substrate were determined by the universal mechanical testing system. Optimized fabrication parameters and their influence on the critical load between sputtered nickel films and polymer substrate were studied by means of the orthogonal experimental design. Increasing radio frequency power and time improved film critical load. The radio frequency power had a more pronounced effect on critical load than the sputter power. The plasma pretreatment with Ar gas modified the surface, leading to an increased surface energy, improving the chemical bonds between nickel and carbon atoms, and thereby enhanced the critical load. The adhesion mechanism is also discussed in this paper.
\end{abstract}

Keywords adhesion, nickel films, plasma treatment, sputtering, surface energy

\section{Introduction}

Metalized plastics have been widely used for products, ranging from car light reflectors, compact disks, pressure or bending sensors, intelligent textiles, robotic interfaces, body sensing devices, and microelectronics (Ref 1-6). Regardless of the application, researchers focus on investigating diffusion and interface formation between metals and polymers, which are quite dissimilar materials. While metals are densely packed crystalline solids with high cohesive energies (Ref 7), polymers contain large macromolecules formed of $10^{2}$ to $10^{5}$ covalently bonded monomeric units, held together by weak van der Waals interactions (Ref 7). Much effort has been made to control the microstructure and thermal stability of metal-polymer interfaces, especially with the aim to improve critical load and to prevent degradation (Ref 8-12). Based on the previous studies, it is obvious that plasma polymer surface modification improves critical load of metal films to polymer substrates (Ref 8). However, only a few studies focus on the relationship between the surface energy, related to the surface modification, and critical load.

In most studies, polycarbonate (PC) was mainly selected as the substrate, because it has pronounced glass transition temperature, and behaves like a typical amorphous polymer.

Xueqiang Qian, Xiaolu Pang, Kewei Gao, and Huisheng Yang, Department of Materials Physics and Chemistry, University of Science and Technology Beijing, Beijing 100083, China; Jie Jin, Beijing Research Institute of Automation for Machinery Industry, Beijing 100053, China; and Alex A. Volinsky, Department of Mechanical Engineering, University of South Florida, Tampa, FL 33620, USA. Contact e-mail: pangxl@mater.ustb.edu.cn.
It is widely used in a variety of applications, ranging from household articles and construction materials, to electronics and optics (Ref 13), specifically in optical data storage, audio compact disks and holographic data storage media (Ref 14). Nickel was selected as a barrier metal layer among other candidates (molybdenum, titanium, copper), since it exhibited outstanding chemical interactions with polymers in previous studies of metal-polymer interfacial reactions (Ref 15).

In this paper, the critical load between sputtered nickel thin films and polycarbonate substrate was investigated as a function of sputtering parameters, including plasma power and time. The critical load of the sputtered nickel films on polycarbonate substrates was measured using a universal mechanical test system. The radio frequency power had the most effect on critical load, thus the surface energy of polycarbonate substrate after plasma treatment was measured using a static contact angle technique. It was found that the critical load of the nickel thin films on the polymer substrate has something to do with the surface activation. By means of the orthogonal experimental design, the optimized fabrication parameters and their effects on the critical load of nickel barrier films were investigated.

\section{Experimental Procedure}

In this study, $2 \mathrm{~mm}$ thick polycarbonate sheets were used as substrates. Polycarbonate substrates were degreased with acetone using an ultrasonic cleaner for $3 \mathrm{~min}$ and further cleaned for $15 \mathrm{~min}$ in an ultrasonic alcohol bath. Finally, the substrates were rinsed with deionized water. PC substrates plasma pretreatment and $\mathrm{Ni}$ deposition were conducted with the parameters listed in Table 1 with sputtering Ar gas pressure of $0.3 \mathrm{~Pa}$. The thickness of nickel films was measured with Dektak 150 stylus profilometer and the results are shown in Table 1. The films structure was characterized using x-ray diffraction (XRD) with $\mathrm{Cu} \mathrm{K} \alpha$ radiation. The critical load of the 
Table 1 Nickel films deposition parameters

\begin{tabular}{|c|c|c|c|c|c|c|c|}
\hline \multirow[b]{2}{*}{ Sample } & \multicolumn{2}{|c|}{ Plasma treatment } & \multicolumn{3}{|c|}{ Plasma deposition } & \multirow[b]{2}{*}{ Critical loads, $\mathbf{N}$} & \multirow[b]{2}{*}{ Contact angles, } \\
\hline & RF power, W & Time, min & RF power, W & Time, min & Thickness, nm & & \\
\hline $\mathrm{J} 1$ & 70 & 15 & 100 & 300 & 1151 & 8.7 & $\ldots$ \\
\hline $\mathrm{J} 2$ & 70 & 15 & 200 & 135 & 1464 & 10.6 & $\ldots$ \\
\hline $\mathrm{J} 3$ & 70 & 15 & 300 & 100 & 1317 & 10.7 & 66.3 \\
\hline $\mathrm{J} 4$ & 70 & 15 & 400 & 75 & 1229 & 12.3 & $\ldots$ \\
\hline J5 & 40 & 15 & 300 & 100 & 1273 & 5.5 & 77.7 \\
\hline J6 & 100 & 15 & 300 & 100 & 1554 & 11.1 & 55.0 \\
\hline $\mathrm{J} 7$ & 70 & 30 & 300 & 100 & 1292 & 10.9 & 59.3 \\
\hline J8 & 70 & 60 & 300 & 100 & 1381 & 13.1 & 41.8 \\
\hline J9 & 100 & 30 & 200 & 135 & 1543 & 11.2 & $\ldots$ \\
\hline
\end{tabular}

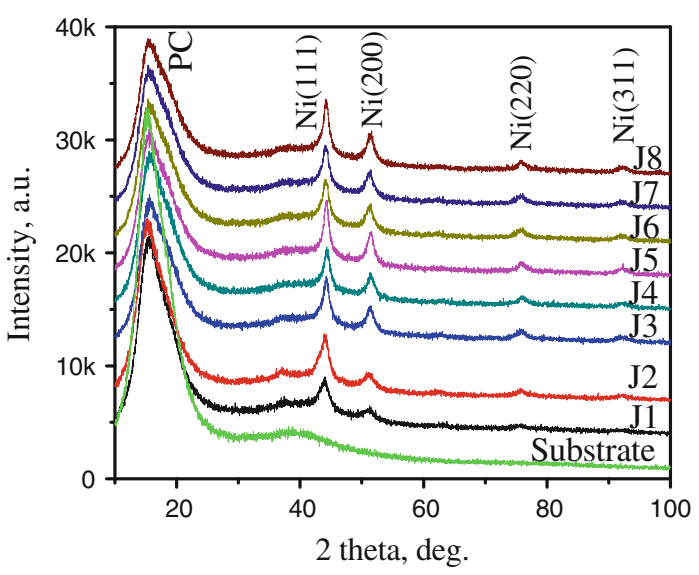

Fig. 1 XRD patterns of Ni films with different sputtering parameters

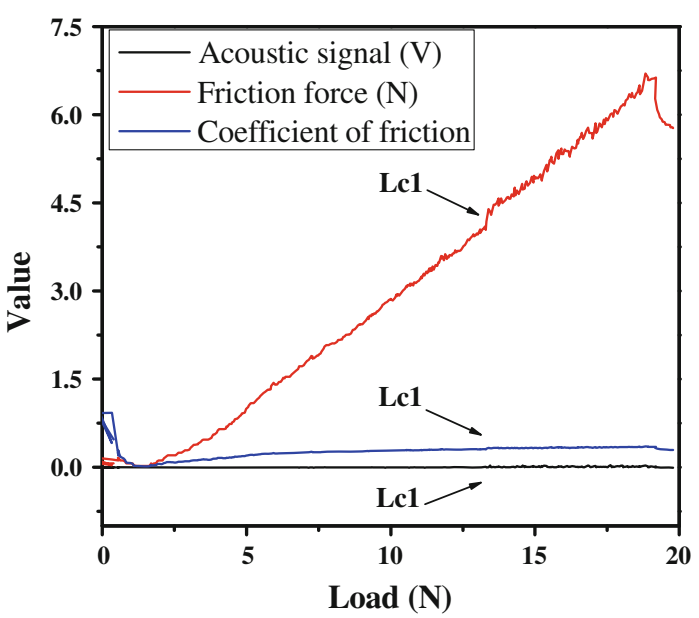

Fig. 2 Acoustic signal, friction force, and friction coefficient vs. the applied load

films was studied with a micro-scratch test instrument (UMT) using a Rockwell diamond indenter with a tip radius of $50 \mu \mathrm{m}$. During the scratch test, the applied load was linearly increased to $20 \mathrm{~N}$, with the $5 \mathrm{~mm} / \mathrm{min}$ scratch velocity, $10 \mathrm{~mm}$ scan length, and $5 \mathrm{~N} / \mathrm{min}$ loading rate. Each sample was tested at least seven times, and the scratches were observed by optical microscopy. Distilled water was chosen to measure the surface energy by means of the contact angel test with different power

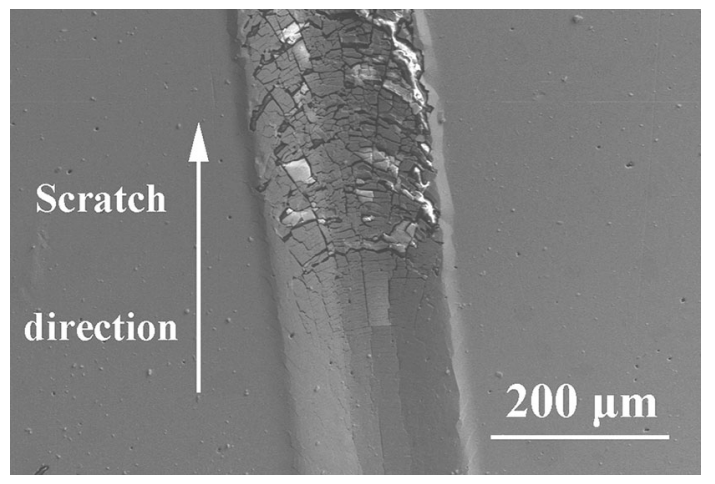

Fig. 3 SEM image of the scratch track at a critical area

and time plasma treatment samples. Water droplets $(3.5 \mu \mathrm{L}$, $1-2 \mathrm{~mm}$ in diameter) were placed vertically from the $3 \mathrm{~mm}$ height with the micro-injector on each sample. Ten measurements were taken, and an average value was reported. All measurements were conducted at room temperature, generally $25^{\circ} \mathrm{C}$. x-Ray photoelectron spectroscopy (XPS, AXIS ULTRA $^{\text {DLD }}$ ) of the substrate surface was performed in vacuum with a monochromated Al K $\alpha$ X-ray source. The source power was $150 \mathrm{~W}$. The binding energy scale was calibrated with respect to the $\mathrm{C} 1 \mathrm{~s}$ peak $(284.8 \mathrm{eV})$. Curve fitting of the spectra was done by a mixture of Gaussian and Lorentzian functions with a Shirley-type background.

\section{Results and Discussion}

Figure 1 shows the typical XRD patterns of uncoated $\mathrm{PC}$ and Ni-coated PC at different deposition parameters. The XRD pattern in Fig. 1 indicated that uncoated PC contains the main amorphous flat peak at around $12^{\circ}$, which is a characteristic peak position for PC (Ref 16). The diffraction peaks at $44.5^{\circ}, 51.6^{\circ}$ $76.2^{\circ}$, and $92.7^{\circ}$ are clearly seen in Fig. 1, besides the weak diffraction peaks of the uncoated PC. It matched with the major (111), (200), (220), and (311) pure Ni reflections, compared with the standard data of JCPDS (65-0380), which reveals that the deposited Ni films coated on PC surface have a face-centered cubic structure. In Fig. 1 the diffraction peaks at $44.5^{\circ}$ and $51.6^{\circ}$ can be clearly seen, but the other reflections are weak. When the sputtering power is raised, the intensity of each XRD peak increases according to these XRD patterns, which implies that the crystallization of Ni films improves with the sputtering power. 


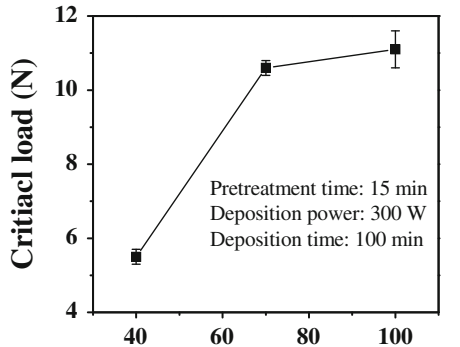

(a) Plasma pretreatment power (W)

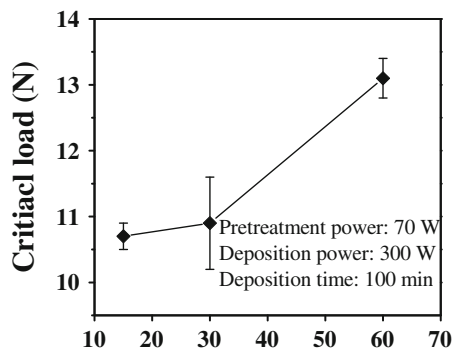

(b) Plasma pretreatment time (min)

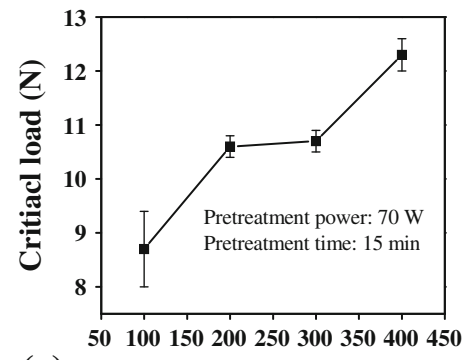

(c) Sputtering power $(\mathrm{W})$

Fig. 4 Critical load dependence on (a) plasma pretreatment power (samples J5, J3, and J6 in Table 1); (b) time (samples J3, J7, and J8 in Table 1), and (c) sputtering power (samples J1, J2, J3, and J4 in Table 1)

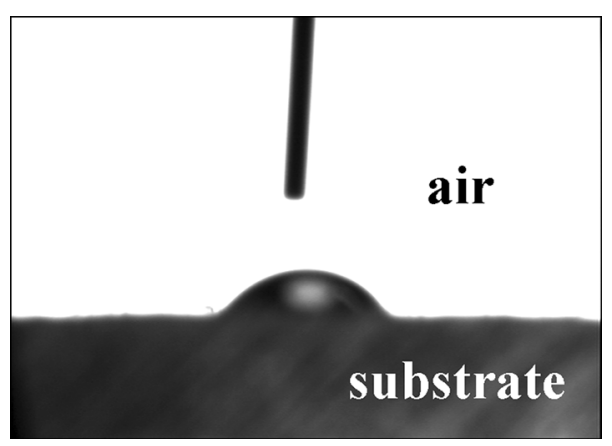

Fig. 5 Contact angle between water droplet and polycarbonate surface in air

The micro-scratch test can be used to evaluate the critical load between the film and the soft plastic substrate presented as critical load (Ref 9). The acoustic emission signal, friction force signal and the coefficient of friction of $\mathrm{Ni}$ films on the polycarbonate substrate are shown in Fig. 2. The data maximum indicates the critical load at which the film breaks, or delaminates from the substrate, allowing assessing its critical load. It is obvious that the acoustic emission, frictional force, and the coefficient of friction changed suddenly when the loading force was about $13.1 \mathrm{~N}$, therefore, this point represents the critical loads of the films.

In this study, the critical load is defined as the load at which failure occurs throughout the film. Figure 3 shows the scratch track of Ni thin film on polycarbonate. The arrow indicates the scratch direction of the indenter tip movement. The first crack on the PC substrate was observed, indicating the cohesive failure between the film and the substrate. There are many cracks near the failure spot, arising from the film interface.

There are three series of samples chosen from Table 1 to show the effects of sputtering parameters on the critical load between the films and the substrate. The J5, J3, and J6 samples were used to investigate the plasma pretreatment power effect on the critical load, as shown in Fig. 4(a), while the J3, J7, and J8 samples were used to investigate the plasma pretreatment time effect on the critical load, as shown in Fig. 4(b). The plasma pretreatment power of the J5, J3, and J6 samples is 40, 70 , and $100 \mathrm{~W}$, and the corresponding critical load of failure is $5.5,10.7$, and $11.1 \mathrm{~N}$, respectively. The plasma pretreatment time of the $\mathrm{J} 3, \mathrm{~J} 7$, and $\mathrm{J} 8$ samples is 15,30 , and $60 \mathrm{~min}$, and the critical load of failure is $10.7,10.9$, and $13.1 \mathrm{~N}$, respectively, as shown in Fig. 4(b). From Fig. 4(a) and (b) it is clear the increasing plasma pretreatment power and time improves film critical load. It is obvious that the substrate modification, resulting from the high dose of radio frequency Ar plasma, yields higher critical load between the Ni films and the PC substrate. In addition, the critical load is correlated with the sputter power (J1, 2, 3, 4) in Fig. 4(c). The critical load increased with higher deposition power, although the critical load increased only slightly at $300 \mathrm{~W}$, compared with $200 \mathrm{~W}$. Ions have more energy at higher sputtering power, so it is possible that $\mathrm{Ni}$ had chemical interactions with $\mathrm{PC}$, which increased the critical load.

In principle, the contact angle of a water droplet on a solid surface in air, as shown in Fig. 5, is determined by the mechanical equilibrium under the action of three interfacial tensions. The relationship between the solid surface energy, $\gamma_{\mathrm{SV}}$, liquid surface energy, $\gamma_{\mathrm{LV}}$, solid-liquid interface interaction energy, $\gamma_{\mathrm{SL}}$, and the equilibrium contact angle, $\theta_{\gamma}$, in solid/ liquid/gas three-phase system is known as the Young's equation:

$\gamma_{\mathrm{SV}}=\gamma_{\mathrm{SL}}+\gamma_{\mathrm{LV}} \cos \theta_{\gamma}$

The Young's equation is based on the following rigorous assumptions: a solid must be smooth, homogeneous and rigid, the solid must not be perturbed by chemical interaction or by adsorption due to a liquid phase, and there should be a single, unique contact angle. It is, however, well known that chemical heterogeneity and surface roughness of practical solid surfaces results in contact angle hysteresis. The advancing contact angle on a smooth, but heterogeneous solid surface, has been regarded as a reasonable estimate of the equilibrium contact angle that would be observed on an ideal surface composed of the low energy surface component (Ref 17).

Owens and Wendt (Ref 18) showed good correlation between surface energies measured by this method and those determined by measuring the contact angles of series of homologous liquids, and those calculated by the Zisman method (Ref 19). Based on these results, surface energy and contact angle have the opposite correlation. The plasma pretreatment power of the J5, J3, and J6 samples is 40,70 , and $100 \mathrm{~W}$, and the contact angle is $77.7^{\circ}$, $66.3^{\circ}, 55.0^{\circ}$, respectively, in Fig. 6(a). The plasma pretreatment time of the J3, J7, and $\mathrm{J} 8$ samples is 15,30 , and $60 \mathrm{~min}$, and the contact angle is $66.3^{\circ}, 59.3^{\circ}$, and $41.8^{\circ}$, respectively, in Fig. 6(b). As the pretreatment power and time increased, the contact angle decreased, and the surface energy increased in Fig. 6(a) and (b). The reduction of the contact angle indicates higher wettability of the surface which could lead to better adhesion at the interface. The critical loads with plasma treatment of substrates presented as contact angles were shown in Fig. 6(c). With the contact angle increasing, the critical load of films and substrates decreases. 


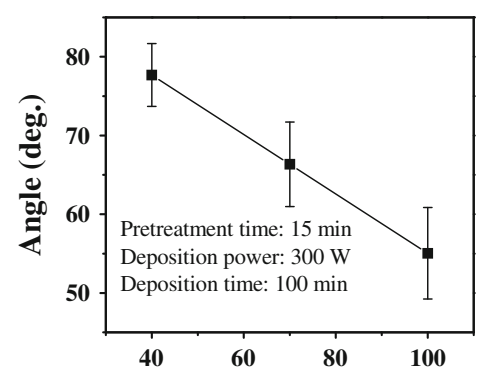

(a) Plasma treatment power (W)

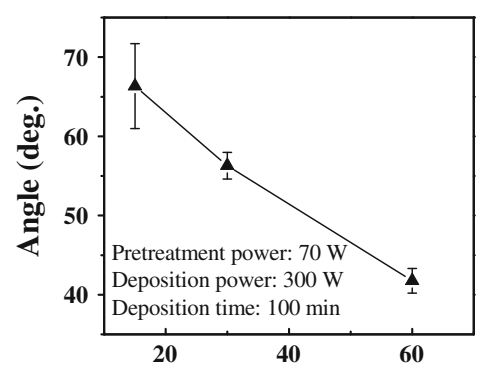

(b) Plasma treatment time (min)

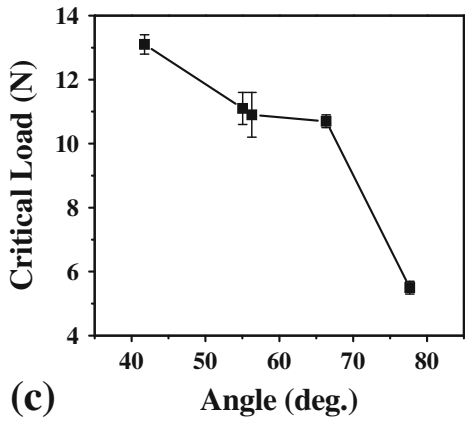

(c)

Angle (deg.)

Fig. 6 Effects of (a) plasma treatment power (samples J5, J3, and J6 in Table 1) and (b) plasma treatment time (samples J3, J7, and J8 in Table 1) on the contact angle of the polycarbonate substrate, (c) critical load of nickel films on polycarbonate as a function of contact angle

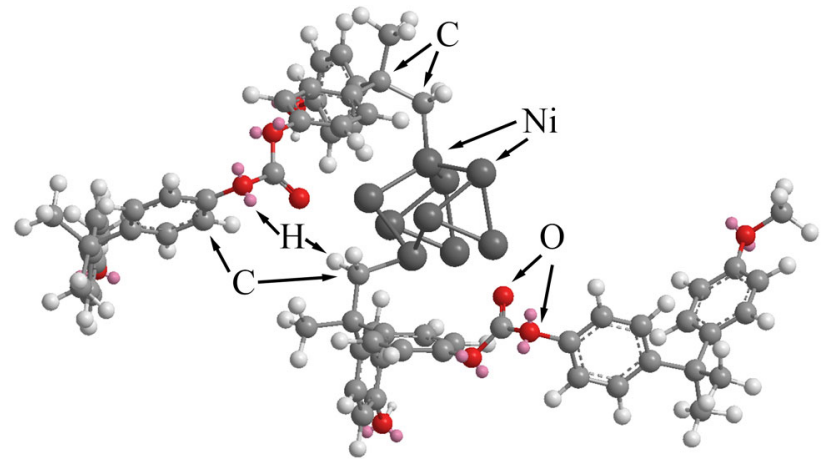

Fig. 7 Three-dimensional model of nickel-polycarbonate interaction mechanisms

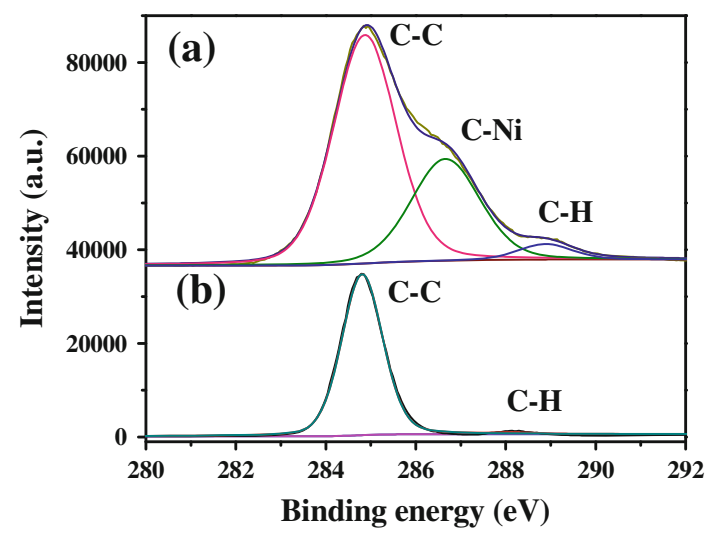

Fig. 8 C1s XPS spectra of the polycarbonate substrate: (a) PC with nickel coating etched in $15 \mathrm{wt} \% \mathrm{HCl}$ solution for $10 \mathrm{~min}$, (b) uncoated PC

During plasma treatment, high energy ions can weaken the van der Waals interactions, creating an open structure, termed "softening of the surface" (Ref 20). There is more space to contain nickel atoms between the macromolecules, inducing covalent bonds between units. Figure 7, created by the ChemBioDraw software, models the polycarbonate chains just under the substrate, perpendicular to the surface. Nickel, which substituted hydrogen in the methyl group for the phenyl structure, is stable. There are face-centered cubic nickel clusters inside the free space between macromolecular chains. The end-group of the macromolecule is exposed to air. There is evidence, by various investigations of polycarbonates, that metals of low reactivity diffuse into polymers during deposition at elevated temperatures, and even form metal clusters inside polycarbonate under such conditions. This mechanism of chemical bonding between metals and polymers was proved by $\mathrm{x}$-ray photoelectron spectroscopy and theoretical analysis (Ref 21, 22).

$\mathrm{x}$-Ray photoelectron spectroscopy was used to prove the mechanism in this experiment. A nickel films with a thickness of $1.2 \mu \mathrm{m}$ was deposited on the polymer substrate with the same deposition conditions as for sample J7. After nickel deposition, the film was etched in $15 \mathrm{wt} . \% \mathrm{HCl}$ solution at room temperature, and the etching was timed to stop at the disappearance of the bubbles. The sample was heat-treated at $100^{\circ} \mathrm{C}$ for $2 \mathrm{~h}$ in air to evaporate water for XPS experiments. Figure 8(a) shows C1s XPS spectra measured form the PC substrate with and without the nickel film to study the adhesion mechanism. Figure 8(b) shows the $\mathrm{C} 1 \mathrm{~s}$ spectrum from a bare $1 \mathrm{~mm}$ thick PC substrate in vacuum. The main peak was calibrated to $284.8 \mathrm{eV}$. The second peak at $288.2 \mathrm{eV}$ corresponds to the ester group in PC, while the main peak can be resolved into three separate peaks: phenyl, methyl and phenol groups (Ref 23). Comparing Fig. 8(a) and (b), there is a significant difference in the spectra. XPS spectrum contains the $\mathrm{C}-\mathrm{Ni}$ bond, recorded at $286.5 \mathrm{eV}$, which proves the existence of chemical bonding between nickel atoms and polycarbonate at the interface (Ref 16). Based on the above discussion, it was recognized that the substrate surface energy reflects the critical load between nickel films and polycarbonate, as the surface energy can be enhanced as a result of the plasma treatment.

In Table 1, one can see that it is beneficial to the critical load of the films to increase the radio frequency power of pretreatment and the sputtering time, which is the same as increasing the sputtering power. Increasing the power can provide the adequate energy to activate the carbon atoms, which act as points to bond metal and polymer. Increasing the pretreatment time can keep the surface active, which is related to the surface energy. Nickel atoms energy is high enough to form chemical bonds, but carbon atoms energy is not high enough, so it was not very effective to change the critical load. From the above discussion, the optimized fabrication parameters, including plasma treatment power, pretreatment and sputtering time, were established. The critical load reached the maximum of $13.1 \mathrm{~N}$ in the range of experimental parameters.

\section{Conclusions}

In this study, the effects of the deposition parameters, including sputtering power, the radio frequency power of Ar, 
and sputtering time, on the critical load between Ni films and the polycarbonate substrates were investigated by the microscratch test. Films grown at higher sputtering power and longer time shows high critical load. In order to strengthen the critical load of sputtered nickel films, the plasma pretreatment with $\mathrm{Ar}$ gas effectively activated the top of the polycarbonate substrates to enhance the surface energy, associated with the contact angle increase. Thus, the surface energy has a direct correlation with critical load. The critical load of Ni films reached the maximum when the pretreatment power was $100 \mathrm{~W}$, with 60 min plasma treatment time, and the $400 \mathrm{~W}$ sputtering powers. The plasma treatment power is the most important factor influencing the critical load between the film and the substrate, because plasma can activate the surface of the PC substrate to induce the chemical bonds between nickel and carbon atoms, improving the critical load.

\section{Acknowledgments}

This work was supported by National Nature Science Foundation of China (51001013, 51271022), Fok Ying Tung Education Foundation (132001), and the Fundamental Research Funds for the Central Universities.

\section{References}

1. P. Livshits, A. Inberg, Y. Shacham-Diamand, D. Malka, Y. Fleger, and Z. Zalevsky, Precipitation of Gold Nanoparticles on Insulating Surfaces for Metallic Ultra-Thin Film Electroless Deposition Assistance, Appl. Surf. Sci., 2012, 258, p 7503-7506

2. S. Hajati, V. Zaporojtchenko, F. Faupel, and S. Tougaard, Characterization of Au Nano-Cluster Formation on and Diffusion Polystyrene Using XPS Peak Shape Analysis, Surf. Sci., 2007, 601, p 3261-3267

3. F. Axisa, P.M. Schmitt, C. Gehin, G. Delhomme, E. McAdams, and A. Dittmar, Flexible Technologies and Smart Clothing for Citizen Medicine, Home Healthcare, and Disease Prevention, IEEE Trans. Inform. Technol. Biomed, 2005, 9, p 325-336

4. T. Someya, T. Sekitani, S. Iba, Y. Katio, H. Kawaguchi, and T. Sakurai, Conformable, Flexible, Large-Area Networks of Pressure and Thermal Sensors with Organic Transistor Active Matrixes, Proc. Natl. Acad. Sci. U.S.A., 2004, 101, p 9966-9970

5. M. Leonardi, E.M. Pitchon, A. Bertsch, Ph. Renaud, and A. Mermoud, Wireless Contact Lens Sensor for Intraocular Pressure Monitoring: Assessment on Enucleated Pig Eyes, Acta Ophthalmol., 2009, 87, p $433-437$
6. R.S. Forrest, The Path to Ubiquitous and Low-Cost Organic Electronic Appliances on Plastic, Nature, 2004, 428, p 911-917

7. R.W. Cahn and P. Haasen, Ed., Physical Metallurgy (in Three Volumes), Elsevier, Amsterdam, 1996

8. E. Dayss, G. Leps, and J. Meinhardt, Surface Modification for Improved Adhesion of a Polymer-Metal Compound, Surf. Coat. Technol., 1999, 116-119, p 986-990

9. L. Sang-Hyuk, P. In-Sun, S. Bo-Hyun, C. Heehwan, J. Jae-Hong, L. Yong Uk, and H. Munpyo, Effect of Sputtering Parameters on the Adhesion of Copper/Molybdenum Metal on Polymer Substrate, Curr. Appl. Phys., 2001, 11, p S12-S15

10. M.J. Cordill, F.D. Fischer, F.G. Rammerstorfer, and G. Dehm, Adhesion Energies of Cr Thin Films on Polyimide Determined from Buckling: Experiment and Model, Acta Mater., 2010, 58, p 5520-5531

11. T. Li and Z. Suo, Ductility of Thin Metal Films on Polymer Substrates Modulated by Interfacial Adhesion, Int. J. Solids Struct., 2007, 44, p 1696-1705

12. C.-H. Jung, H.-W. Cho, I.-T. Hwang, J.-H. Choi, Y.-C. Nbo, J.-S. Shin, and K.-H. Chang, The Effects of Energetic Ion Irradiation on Metal-toPolymer Adhesion, Radiat. Phys. Chem., 2012, 81-8, p 919-922

13. J.T. Bendler, C.A. Edmondson, M.C. Wintersgill, D.A. Boyles, T.S. Filipova, and J.J. Fontanella, Electrical Properties of a Novel Fluorinated Polycarbonate, Eur. Polymer J., 2012, 48-4, p 830-840

14. M. Toishi, T. Tanaka, A. Fukumoto, M. Sugiki, and K. Watanade, Evaluation of Polycarbonate Substrate Hologram Recording Medium Regarding Implication of Birefringence and Thermal Expansion, Opt. Coтmun., 2007, 270-1, p 17-24

15. F. Faupel, R. Willeckem, and A. Thran, Diffusion of Metals in Polymers, Mater. Sci. Eng., 1998, R22, p 1-55

16. A.K. Singh, R.K. Mishra, R. Prakash, P. Maiti, A.K. Singh, and D. Pandey, Specific Interactions in Partially Miscible Polycarbonate (PC)/ Poly(methyl methacrylate) (PMMA) Blends, Chem. Phys. Lett., 2010, 486, p 32

17. E. Oh and P.E. Luner, Surface Free Energy of Ethylcellulose Films and the Influence of Plasticizers, Int. J. Pharm., 1999, 188, p 203-219

18. D.K. Owens and R.C. Wendt, Estimation of the Surface Free Energy of Polymers, J. Appl. Polym. Sci., 1969, 13, p 1741-1747

19. W.A. Zisman, Influence of Constitution on Adhesion, Ind. Eng. Chem., 1963, 55, p 18-38

20. R.L.W. Smithson, D.J. McClure, and D.F. Evans, Effects of Polymer Substrate Surface Energy on Nucleation and Growth of Evaporated Gold Films, Thin Solid Films, 1997, 307, p 110-112

21. L.P. Kazansky, I.A. Selyaninov, and Yu.I. Kuznetsov, Angle Resolved XPS of Monomolecular Layer of 5-Chlorobenzotriazole on Oxidized Metallic Surface, Appl. Surf. Sci., 2012, 259, p 385-392

22. M. Mortazavi and M. Nosonovsky, A Model for Diffusion-Driven Hydrophobic Recovery in Plasma Treated Polymers, Appl. Surf. Sci., 2012, 258, p 6876-6883

23. A. Chenite, A. Selmani, and A. Yelon, X-ray Photoelectron Spectroscopy Investigation of Interfacial Interactions in the Cr/BPDA-PDA and Ti/BPDA-PDA Systems, J. Vac. Sci. Technol. A, 1994, 12, p 513-522 